\title{
DIGITALCOMMONS
}

$5-1-2008$

\section{On the Length of NHL Shootouts}

W.J. Hurley

Royal Military College of Canada, hurley-w@rmc.ca

Follow this and additional works at: http://digitalcommons.wayne.edu/jmasm

Part of the Applied Statistics Commons, Social and Behavioral Sciences Commons, and the Statistical Theory Commons

\section{Recommended Citation}

Hurley, W. J. (2008) "On the Length of NHL Shootouts," Journal of Modern Applied Statistical Methods: Vol. 7 : Iss. 1 , Article 27. DOI: $10.22237 /$ jmasm/1209615960

Available at: http://digitalcommons.wayne.edu/jmasm/vol7/iss1/27 


\title{
Brief Reports \\ On the Length of NHL Shootouts
}

\author{
W. J. Hurley \\ Royal Military College of Canada
}

When NHL teams are tied after 60 minutes of regulation time and 5 minutes of sudden-death overtime, they go to a shootout to determine who gets the overtime point. Teams alternate shots until a winner is determined. The probability of observing shootouts of various lengths is calculated.

Key Words: probability tree, independent trials, Hockey shootout

\section{Introduction}

It is certainly true that statisticians have a fascination with records, particularly with sports records. The interested reader is referred to Nevzorov (2001) for a comprehensive treatment of the statistical theory associated with records. The primary motivation in this literature is the prediction of the way records will change over time. Noubary (2005) is illustrative of this genre.

When NHL teams are tied after 60 minutes of regulation time and 5 minutes of sudden-death overtime, they go to a shootout to determine who gets the overtime point. Teams alternate shots until a winner is determined. The shootout went 26 shots in a game between the Flyers and Rangers during the 2006-2007 season. The NHL record is 30 shots. Given the sudden death structure of shootouts, it seemed these ought to be rare events.

\section{NHL Shootout Rules}

The shootout is governed by Rule 89 (Note 4) of the NHL Rulebook: "Each team will be given three shots, unless the outcome is determined earlier in the shootout. After each team has taken three shots, if the score remains tied, the shootout will proceed to a "sudden

William Hurley is a Professor in the Department of Business Administration at the Royal Military College of Canada. His research interests are military operations research, decision analysis, and game theory. E-mail: hurley-w@rmc.ca. death" format. No player may shoot twice until everyone who is eligible has shot."

Rule 89 can be found at: http://www.nhl.com/rules/rule89.html. As Rule 89 suggests, it is possible to complete the shootout in fewer than six shots. For instance, if the team shooting first scores on its first two shots and the other team misses on its first two, there is no need for the teams to take their third shots. Once the shootout gets to the "sudden death" portion, the teams effectively generate a sequence of pairs of shots. The first pair comprises shots 7 and 8, the second, shots 9 and 10 , and so on. Note that the shootout will proceed to pair $n+1$ shots if either both teams score on their pair $n$ shots or both teams do not score on their pair $n$ shots.

\section{Shootout Statistics}

Table 1 shows the frequencies of shootout lengths for the complete 2005-2006 season and the 2006-2007 season up to midNovember, 2006. In total, 176 games went to a shootout. Of these, 56 went to the sudden death format. Of some importance to the calculation are the probabilities that teams score on a shot. Over the short history of the shootout to date, 1,241 shots have been taken, and of these, 408 were successful. The frequency of scoring a goal, then, is

$$
\frac{408}{1241}=0.329
$$




\section{W. J. HURLEY}

Table 1.

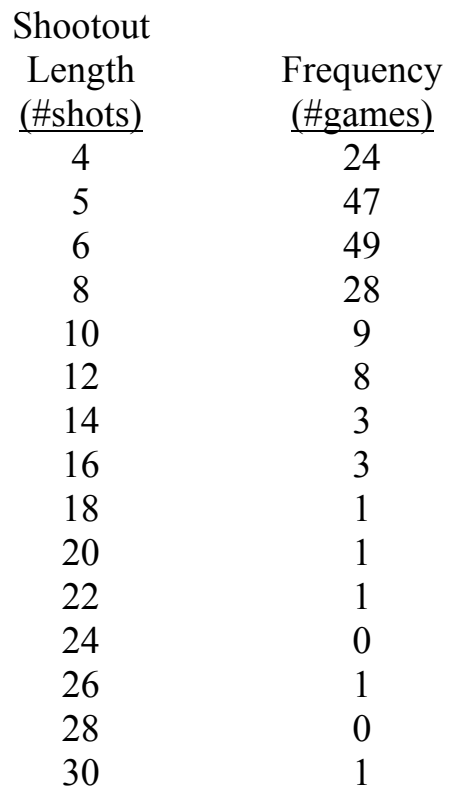

First 6 shooters

All other shooters

Totals

Table 2.

\begin{tabular}{|c|c|c|}
\hline \#Shots & \#Goals & Frequency \\
\hline 961 & 324 & 0.337 \\
\hline 280 & 84 & 0.300 \\
\hline 1,241 & 408 & 0.329 \\
\hline
\end{tabular}

It is interesting to note that this frequency is comparable to the chance of hitting a Major League pitcher, a task generally considered to be one of the toughest in all of sport.

An obvious assumption is that all shots are independent trials and that each shooter has a probability, $p$, of scoring. However this ignores the fact that coaches will order shooters according to their ability to score. Hence it is probably the case that players shooting earlier are more likely to score. To assess these relative probabilities of scoring, I arbitrarily partitioned shooters into those taking the first 6 shots and those taking the rest of the shots. Table 2 shows their relative performance over 1,241 shots. Note that the first 6 shooters have a higher frequency of scoring. However the difference is not enough to reject the null that both groups have the same probability of scoring.

Analysis

The first step is to calculate the probabilities of various length shootouts for the first 6 shots. Let $p$ be the probability that a shooter scores. I assume this probability applies to each of the first three shooters on both teams. Let $L_{n}$ be the probability that the length of the shootout is exactly $\mathrm{n}$ shots. Evaluating a probability tree, the following formulae are obtained. The probability of the shootout going to sudden death is 


\section{ON THE LENGTH OF NHL SHOOTOUTS}

Table 2. Probability of Shootout Going to Sudden Death

\begin{tabular}{|c|c|c|}
\hline Length & Uniform & Non-uniform \\
\hline 4 & 0.0974 & 0.1000 \\
\hline 5 & 0.2466 & 0.2472 \\
\hline 6 & 0.3185 & 0.3180 \\
\hline 8 & 0.1490 & 0.1406 \\
\hline 10 & 0.0832 & 0.0815 \\
\hline 12 & 0.0465 & 0.0473 \\
\hline 14 & 0.0260 & 0.0274 \\
\hline 16 & 0.0145 & 0.0159 \\
\hline 18 & 0.0081 & 0.0092 \\
\hline 20 & 0.0045 & 0.0054 \\
\hline 22 & 0.0025 & 0.0031 \\
\hline 24 & 0.0014 & 0.0018 \\
\hline 26 & 0.0008 & 0.0010 \\
\hline 28 & 0.0004 & 0.0006 \\
\hline 30 & 0.0002 & 0.0004 \\
\hline
\end{tabular}

$$
\begin{aligned}
L_{4} & =2 p^{2}(1-p)^{2} \\
L_{5} & =2 p(1-p)\left[p^{2}+(1-p)^{2}\right] \\
L_{6} & =4 p(1-p)\left\{\left[p^{2}+(1-p)^{2}\right]^{2}+p^{2}(1-p)^{2}\right\} \\
S_{p} & =\left[p^{2}+(1-p)^{2}\right]\left[\left[p^{2}+(1-p)^{2}\right]^{2}+6 p^{2}(1-p)^{2}\right\} .
\end{aligned}
$$

Once in sudden death, the length of the shootout follows a geometric distribution. Note that $p^{2}+(1-p)^{2}$ is the probability that the shooters either both score or both miss and $2 p(1-p)$ is the probability that one scores and the other misses. Hence, the probability the shootout goes $m$ shots for values of $m$ at least 8 is

$$
\begin{aligned}
& L_{m}=S_{p}\left[p^{2}+(1-p)^{2}\right]^{m / 2-4} 2 p(1-p) \\
& \text { for } m=8,10,12, \ldots
\end{aligned}
$$

In the case where the first six shooters have a probability $p$ of scoring and those shooting in the sudden death portion have a probability $\bar{p}$ of scoring, $L_{m}$ is modified to

$$
L_{m}=2 S_{p} \bar{p}(1-\bar{p})\left[\bar{p}^{2}+(1-\bar{p})^{2}\right]^{m / 2-4}
$$

for $m=8,10,12, \ldots$

The probabilities of shootout length for two assumptions about the underlying probabilities of scoring on a single shot are calculated by:

1. Uniform: All shooters score with probability $p=0.329$.

2. Non-uniform: The first six shooters score with probability $p=0.337$ and all other shooters score with probability $\bar{p}=0.300$.

Table 3 gives the densities for lengths up to 30 shots. Not surprisingly the two distributions are close and the tail of the Non-uniform density is slightly larger.

Using the Non-uniform distribution, the probability of a shootout going at least 20 shots is 0.013 . Of the 176 shootout games, 4 have gone at least 20 shots and the $p$-value of this outcome (the chance of observing 4 or more games taking at least 20 shots) is 0.19 . Hence what actually happened is consistent with the theoretical prediction. 


\section{W. J. HURLEY}

Another interesting question is the time between rare events. For instance, the average time between shootouts of at least 40 shots can be calculated. If the experience to date with penalty shots continues then the argument proceeds as follows. Using the Non-uniform probabilities, the chance of observing a shootout going at least 40 shots is 0.00005489 . Assuming 1,230 NHL games a year and that, for each of these, there is a $12 \%$ chance that a game will get to a shootout, the average number of seasons between 40 -shot shootouts is

$$
\frac{1}{1230 \times .12 \times .00005489}=123.4 \text { seasons }
$$

Equivalently, there is about a $50 \%$ chance of observing a shootout going at least 40 shots in the next 125 years.

\section{References}

Nevzorov, V. B. (2001). Records: mathematical theory. American Mathematical Society. Providence, RI.

Noubary, R. D. (2005). A procedure for prediction of sports records. Journal of Quantitative Analysis in Sports, 1(4). Available at http://www.bepress.com/jqas/vol1/iss1/4. 